\title{
Improvements in the X-ray luminosity function and constraints on the cosmological parameters from $\mathrm{X}$-ray luminous clusters
}

\author{
A. Del Popolo ${ }^{1,2}$, V. Costa ${ }^{3}$, and G. Lanzafame ${ }^{4}$
}

\author{
1 Dipartimento di Fisica e Astronomia, Università di Catania, Viale Andrea Doria 6, 95125 Catania, Italy \\ e-mail: antonino.delpopolo@unibg.it \\ 2 Argelander-Institut für Astronomie, Auf dem Hügel 71, 53121 Bonn, Germany \\ 3 Dipartimento di Metodologie Chimiche e Fisiche per l'Ingegneria, Univesità di Catania, Viale A. Doria 6, 95125, Catania, Italy \\ ${ }^{4}$ Osservatorio Astrofisico di Catania, Istituto Nazionale di Astrofisica, via S. Sofia 78, 95123, Catania, Italy
}

Received 17 August 2009 / Accepted 31 December 2010

\begin{abstract}
Aims. We improve the current constraints on $\Omega_{\mathrm{m}}$, the dark-energy equation-of-state parameter, $w$, and $\sigma_{8}$, obtained from measurements of the X-ray luminosity function of galaxy clusters, namely MACS, the local BCS, and the REFLEX galaxy cluster samples with luminosities $L>3 \times 10^{44} \mathrm{erg} / \mathrm{s}$ in the $0.1-2.4 \mathrm{keV}$ band.

Methods. To this aim, we use Tinker and collaborators mass function instead of Jenkins and collaborators and the mass-luminosity relationship obtained by Del Popolo and collaborators.

Results. Using the same methods and priors as Mantz and collaborators, we find $\Omega_{\mathrm{m}}=0.28_{-0.04}^{+0.05}$ and $\sigma_{8}=0.78_{-0.05}^{+0.04}$, for a $\Lambda \mathrm{CDM}$ universe, while the result of Mantz and collaborators gives less tight constraints $\Omega_{\mathrm{m}}=0.28_{-0.07}^{+0.11}$ and $\sigma_{8}=0.78_{-0.13}^{+0.11}$. In the case of a $w \mathrm{CDM}$ model, we find $\Omega_{\mathrm{m}}=0.27_{-0.06}^{+0.07}, \sigma_{8}=0.81_{-0.06}^{+0.05}$ and $w=-1.3_{-0.4}^{+0.3}$, while in Mantz and collaborators they are again less tight $\Omega_{\mathrm{m}}=0.24_{-0.07}^{+0.15}, \sigma_{8}=0.85_{-0.20}^{+0.13}$ and $w=-1.4_{-0.7}^{+0.4}$. Combining the XLF analysis with the $f_{\text {gas }}+\mathrm{CMB}+\mathrm{SNIa}$ data set results in the constraint $\Omega_{\mathrm{m}}=0.269 \pm 0.012, \sigma_{8}=0.81 \pm 0.021$ and $w=-1.02 \pm 0.04$, to be compared with Mantz and collaborators, $\Omega_{\mathrm{m}}=0.269 \pm 0.016, \sigma_{8}=0.82 \pm 0.03$ and $w=-1.02 \pm 0.06$. The tightness of the last constraints obtained by Mantz and collaborators, are fundamentally due to the tightness of the $f_{\text {gas }}+\mathrm{CMB}+\mathrm{SNIa}$ constraints and not to their XLF analysis. Our findings, consistent with $w=-1$, lend additional support to the cosmological-constant model.
\end{abstract}

Key words. cosmology: theory - cosmological parameters

\section{Introduction}

Clusters of galaxies are the largest gravitationally-collapsed structures in the Universe. Even at the present epoch they are relatively rare, with only a few percent of all galaxies in clusters. In the hierarchical collapse scenario for structure formation in the universe, the number density of collapsed objects as a function of mass and cosmic time is a sensitive probe in cosmology. The galaxy clusters that occupy the high-mass tail of this population provide a powerful and relatively clean tool, since their growth is predominantly determined by linear gravitational processes. Starting from the 1990s, analyses of massive clusters have consistently indicated low values for $\Omega_{\mathrm{m}}$, from the baryonic fraction arguments (White et al. 1993) and measurements of the evolution in the cluster number density (Eke et al. 1998; Borgani et al. 2001), and from low values of $\sigma_{8}{ }^{1}$ (Henry \& Arnaud 1991; Reiprich \& Böringer 2002; Schuecker et al. 2003). A result later confirmed by cosmic microwave background (CMB) studies, cosmic shear, and other experiments (Spergel et al. 2007; Komatsu et al. 2008; Dunkley et al. 2008; Benjamin et al. 2007; Fu et al. 2008). For precision's sake, cluster surveys in the local universe are particularly useful for constraining a combination

\footnotetext{
${ }^{1} \sigma_{8}$ is the amplitude of the mass density fluctuation power spectrum over spheres of radius $8 h^{-1} \mathrm{Mpc}$, and $M_{8}$ is the mean mass within these spheres.
}

of the matter density parameter $\Omega_{\mathrm{m}}$ and the normalization of the power spectrum of density fluctuations. Following the evolution of the cluster space density over a wide redshift baseline, one can break the degeneracy between $\sigma_{8}$ and $\Omega_{\mathrm{m}}$ (Rosati et al. 2002).

Recently, X-ray studies (Vikhlinin et al. 2009b) of the evolution of the cluster mass function at $z=0-0.8$ have convincingly demonstrated that the growth of cosmic structure has slowed down at $z<1$ owing to the effects of dark energy. Thus such measurements have been used to improve its equation of state parameter. Although the quoted cosmological test is very powerful, there are two main problems for practical applications: first, theoretical predictions provide the number density of clusters of a given mass, while the mass itself is never the directly observed quantity. Second, a cluster sample is needed that spans a wide$z$ baseline and is based on model-independent selection criteria ${ }^{2}$.

Determining the evolution of the space density of clusters requires counting the number of clusters of a given mass per unit volume at different redshifts. Therefore, three essential tools are required for its application as a cosmological test: (a) an efficient method of finding clusters over a wide redshift range; (b) an observable estimator of the cluster mass; and (c) a method of computing the selection function or, equivalently, the survey volume

\footnotetext{
2 This is so that the search volume and the number density associated with each cluster are uniquely identified.
} 
within which clusters are found. Observations of clusters in the $\mathrm{X}$-ray band provide an efficient and physically motivated method of identification, which fulfills the three requirements above. The X-ray luminosity, $L_{\mathrm{x}}$, provides a very efficient method for identifying clusters down to a given X-ray flux limit so that, within a known survey volume for each $L_{\mathrm{x}}$, which uniquely specifies the cluster selection, it is also a good probe of the depth of the cluster gravitational potential.

For these reasons most of the cosmological studies based on clusters have used X-ray-selected samples. According to the three points quoted above, the recipe for constraining cosmological parameters by means of clusters is composed of three ingredients:

1) the predicted mass function of clusters, $n(M, z)$, as a function of cosmological parameters $\left(\sigma_{8}, \Omega_{\mathrm{m}}, w\right.$, etc. $)$;

2) sky surveys with well-understood selection functions to find clusters, as well as a relation linking cluster mass with an observable. A successful solution to the former requirement has been to identify clusters by the X-ray emission produced by hot intracluster gas, notably using data from ROSAT ${ }^{3}$;

3) tight and well-determined scaling relation between survey observable (e.g. $L_{\mathrm{x}}$ ) and mass, with minimal intrinsic scatter.

Early attempts to use the evolution of the cluster mass function as a cosmological probe were limited by small sample sizes and by either poor proxies for the cluster mass (e.g., the total X-ray flux) or inaccurate measurements (e.g. temperatures with large uncertainties).

Until some years ago, the results for $\Omega_{\mathrm{m}}$ disagree several times. Studies by different authors (Bahcall et al. 1997; Bahcall \& Fan 1998; Sadat et al. 1998; Blanchard et al. 1998; Blanchard \& Bartlett 1998; Eke et al. 1998; Viana \& Liddle 1999; Reichart et al. 1999; Donahue \& Voit 1999; Borgani et al. 2001) found values for $\Omega_{\mathrm{m}}$ spanning the entire range of acceptable values: $0.2 \leq \Omega_{\mathrm{m}} \leq 1$. On the basis of the same data set of Eke et al. (1998) with Viana \& Liddle (1999), showed that uncertainties both in fitting local data and in the theoretical modelling could significantly change the final results. They found $\Omega_{\mathrm{m}} \simeq 0.75$ as a preferred value with a critical density model acceptable at $<90 \%$ c.l., while Eke et al. (1998) found $\Omega_{\mathrm{m}}=0.45 \pm 0.2$.

The reasons leading to the quoted discrepancies have been studied in several papers (Eke et al. 1998; Reichart et al. 1999; Donahue \& Voit 1999; Borgani et al. 2001) and can be summarized as coming from: 1) the inadequate approximation given by the mass function used (e.g., Bryan \& Norman 1998); 2) inadequacy in the structure formation as described by the spherical model leading to changes in the threshold parameter $\delta_{\mathrm{c}}$ (e.g., Governato et al. 1999); 3) inadequacy in masstemperature relation $(M-T)$ obtained from the virial theorem (see Voit \& Donahue 1998; Del Popolo 2002); 4) effects of cooling flows; 5) determination of the X-ray cluster catalog's selection function; 6) missing high redshift clusters in the data used

\footnotetext{
3 The ROSAT Brightest Cluster Sample (BCS; Ebeling et al. 1998, 2000) and ROSAT-ESO Flux Limited X-ray sample (REFLEX; Böhringer et al. 2004) together cover approximately two-thirds of the sky out to redshift $z \simeq 0.3$ and contain more than 750 clusters. The Massive Cluster Survey (MACS; Ebeling et al. 2001, 2007) extends these data to $z \simeq 0.7$. The ROSAT 160 sq. degree survey, described for the first time by Vikhlinin et al. (1998) is a serendipitous cluster catalogue containing 201 groups/clusters, while the ROSAT 400 sq. degree survey is based on 1610 high Galactic latitude ROSAT PSPC pointings (Burenin et al. 2007) and includes 266 optically confirmed galaxy clusters, groups and individual elliptical galaxies.
}

(e.g., the $E M S S)$; 7) evolution of the luminosity-temperature relation $(L-T)$ (Voit \& Donahue 1998); 8) use of different bestfitting procedures to get the constraints (Eke et al. 1998); 9) other effects described in more recent papers (e.g., Mantz et al. 2008, hereafter M08; Vikhlinin et al. 2009b).

The situation with the cluster mass function data has been dramatically improved in past years. A large sample of sufficiently massive clusters extending to $z \simeq 0.9$ has been derived from ROSAT PSPC pointed data covering $400 \mathrm{deg}^{2}$ (Burenin et al. 2007). Distant clusters from the 400d sample were then observed with Chandra, providing high-quality X-ray data and much more accurate total mass indicators (see Vikhlinin et al. 2009b). Chandra coverage has also become available for a complete sample of low- $z$ clusters originally derived from the ROSAT All-Sky Survey (see Vikhlinin et al. 2009b). Results from deep Chandra pointings to a number of low- $z$ clusters have significantly improved our knowledge of the outer cluster regions and provided a much more reliable calibration of the $M_{\text {tot }}$ vs. proxy relations than was possible before. On the theoretical side, improved numerical simulations resulted in better understanding of measurement biases in the X-ray data analysis (Nagai et al. 2007; Rasia et al. 2006; Jeltema et al. 2007). In the present paper, we want to show how tighter constraints can be obtained in the M08 model, improving the mass function adopted by them, and the scaling laws used (e.g., the $M-T$ and Mass-Luminosity $(M-L)$ relationships). In this paper, we use the observed X-ray luminosity function to investigate two cosmological scenarios, assuming a spatially flat metric in both cases: the first includes dark energy in the form of a cosmological constant $(\Lambda \mathrm{CDM})$, and the second has dark energy with a constant equation-of-state parameter, $w(w \mathrm{CDM})$. The theoretical background for this work is reviewed in Sect. 2. Section 3 presents the results and Sect. 4 the conclusions.

\section{Theory}

After discussing the ingredients needed in the recipe used to constrain cosmological parameters from X-ray observations, now we derive an expression for the X-ray luminosity function (XLF) (using now the mass function obtained in Del Popolo 2006a,b and $M-T, L-T$ relations obtained in Del Popolo 2002 and Del Popolo et al. 2005, respectively) and then set some constraints on $\Omega_{\mathrm{m}}, \sigma_{8}$, and the dark-energy equation-of-state parameter $w$, by using the data (clusters) used in M08, namely MACS (Massive Cluster Survey), BCS (Brightest Cluster Sample), and REFLEX (ROSAT ESO FLUX LIMITED X-ray SAMPLE). Following M08, the constraints are obtained from measurements of the XLF of the quoted samples. The most straightforward mass-observable relation to complement these X-ray flux-limited surveys is the mass-X-ray luminosity relation. For sufficiently massive (hot) objects at the relevant redshifts, the conversion from X-ray flux to luminosity is approximately independent of temperature, in which case the luminosities can be estimated directly from the survey flux and the selection function is identical to the requirement for detection. A disadvantage is that there is large scatter in cluster luminosities at fixed mass; however, having enough data allows this scatter to be quantified empirically. More recently, a dramatic reduction in luminositymass scatter has been demonstrated when luminosities are measured by excluding cluster centres (typically $r<0.15 r_{500}$; Maughan 2007; Zhang et al. 2007). Alternative approaches use cluster temperature (Henry 2000; Seljak 2002; Pierpaoli et al. 2003; Henry 2004), gas fraction (Voevodkin \& Vikhlinin 2004) or $Y_{X}$ parameter (Kravtsov et al. 2006) to achieve tighter 
mass-observable relations at the expense of reducing the size of the samples available for analysis. The need to quantify the selection function in terms of both X-ray flux and a second observable additionally complicates these efforts.

The first ingredient of the quoted recipe (i.e., mass function) used in M08 was the Jenkins et al. (2001, hereafter J01) mass function. J01 writes the mass function of galaxy clusters of mass $M$ at redshift $z$ as a "universal function" of $\sigma^{-1}(M, z)$

$f\left(\sigma^{-1}\right)=\frac{M}{\rho_{\mathrm{m}}} \frac{n(M, z)}{\mathrm{d} \ln \sigma^{-1}}$

which is fitted by

$f\left(\sigma^{-1}\right)=A \mathrm{e}^{\left(-\left|\ln \sigma^{-1}+B\right|^{\epsilon}\right)}$

for cosmological -constant models, with $A=0.316, B=0.67$, and $\epsilon=3.82$.

As shown in Del Popolo (2006a,b), the theoretical mass function obtained in the quoted papers is in better agreement with high-resolution $N$-body simulations, namely Reed et al. 2003 (R03), Yahagi et al. (2004) (YNY), Warren et al. 2006 (W06), and Tinker et al. (2008, see the following and Fig. 1b) (T08).

The mass function is calculated according to the model of Del Popolo (2006a,b). The multiplicity function, in the quoted model, is given by

$$
\begin{aligned}
v f(v)= & A_{1}\left(1+\frac{\beta_{1} g\left(\alpha_{1}\right)}{(a v)^{\alpha_{1}}}+\frac{\beta_{2} g\left(\alpha_{2}\right)}{(a v)^{\alpha_{2}}}+\frac{\beta_{3} g\left(\alpha_{3}\right)}{(a v)^{\alpha_{3}}}\right) \\
& \times \sqrt{\frac{a v}{2 \pi}} \mathrm{e}^{\left\{\frac{-a v}{2}\left[1+\frac{\beta_{1}}{(a v)^{\alpha_{1}}}+\frac{\beta_{2}}{(a v)^{\alpha_{2}}}+\frac{\beta_{3}}{(a v)^{\alpha_{3}}}\right]^{2}\right\}}
\end{aligned}
$$

where

$$
\begin{aligned}
g\left(\alpha_{i}\right)= & \mid 1-\alpha_{i}+\frac{\alpha_{i}\left(\alpha_{i}-1\right)}{2 !} \\
& -\ldots-\frac{\alpha_{i}\left(\alpha_{i}-1\right) \cdots\left(\alpha_{i}-4\right)}{5 !} \mid
\end{aligned}
$$

where $i=1$ or $2, \alpha_{1}=0.585, \beta_{1}=0.46, \alpha_{2}=0.5$ and $\beta_{2}=$ $0.35, \alpha_{3}=0.4$ and $\beta_{3}=0.02, a=0.707$, and $A_{1}=1.2$ is the normalization constant.

The "multiplicity function" is correlated with the usual, more straightforwardly used, "mass function" as follows. Following Sheth \& Tormen (2002, hereafter ST) notation, if $f(M, \delta) \mathrm{d} M$ denotes the fraction of mass that is contained in collapsed haloes that have mass in the range $M-M+\mathrm{d} M$, at redshift $z$, and $\delta(z)$ is the redshift dependent overdensity, the associated "unconditional" mass function is

$n(M, \delta) \mathrm{d} M=\frac{\rho_{\mathrm{b}}}{M} f(M, \delta) \mathrm{d} M$.

In Fig. 1a, we plot the multiplicity function obtained in this paper (symbols are described in the figure caption).

There are some differences between the quoted simulations and the J01 simulations. First, the multiplicity function of the present paper, similar to that of YNY, in the low- $v$ region of $v \leq 1$ systematically falls below the J01 functions. In this region the multiplicity function of the present paper is very close to that of YNY. Additionally, the numerical multiplicity functions (and that in Del Popolo 2006a,b) have an apparent peak at $v \simeq$ instead of the plateau that is seen in the J01 function. Similar differences are seen in the high- $v$ region. These differences between

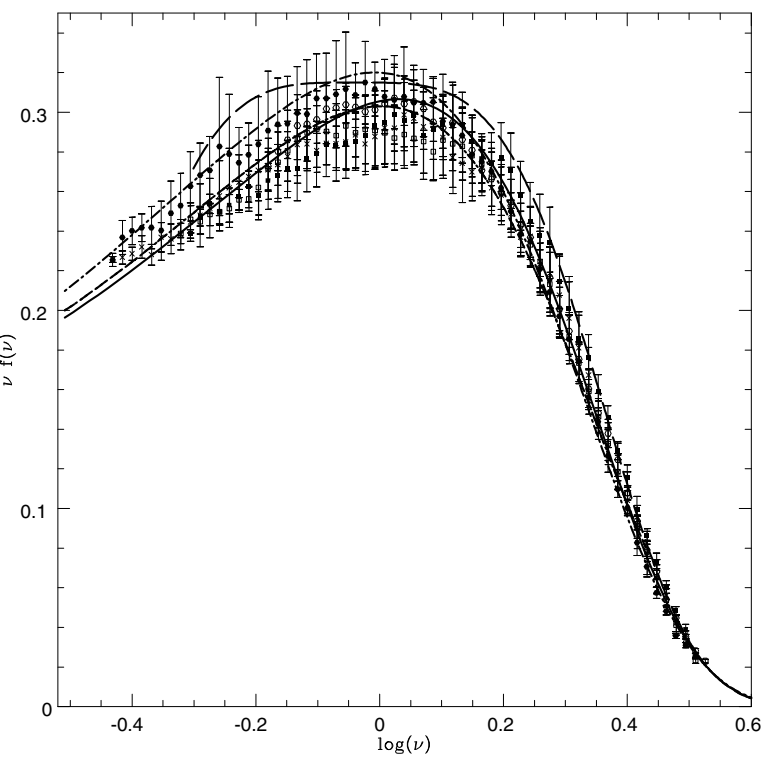

(a)

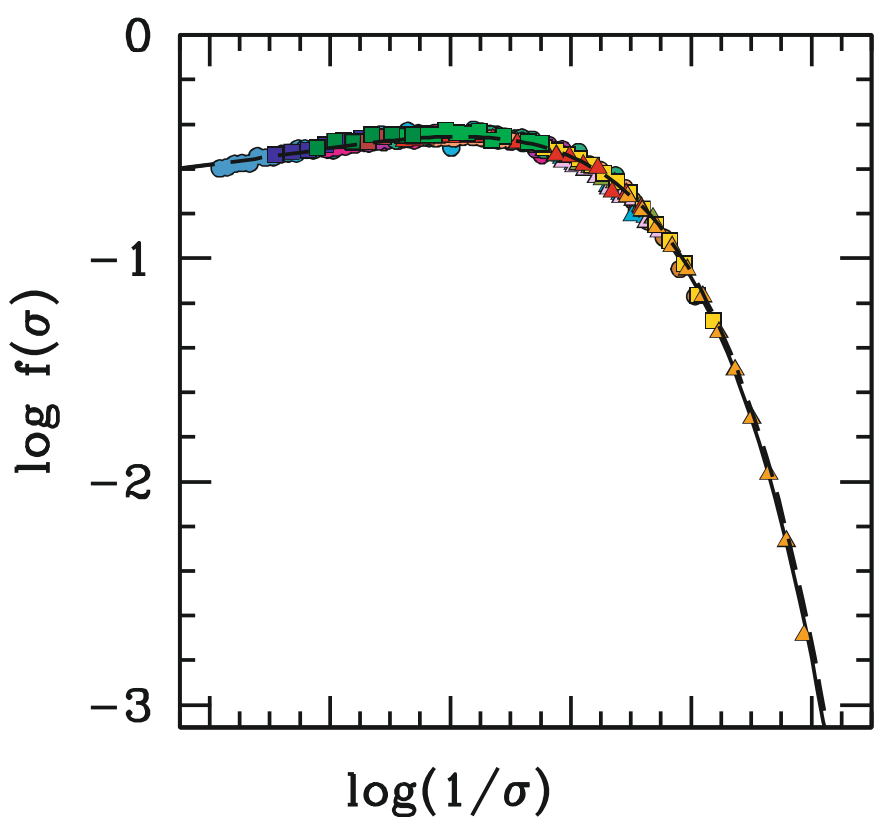

(b)

Fig. 1. In Panel a): multiplicity function obtained in this paper (solid line), fitting formula proposed by Yahagi et al. (2004) their Eq. (7) (short-dashed line), Sheth \& Tormen (2002) (ST) multiplicity function (dotted line), Jenkins et al. (2001) multiplicity function (long-dashed line). The error bars with open circles represent the run 140 of YNY, those with filled squares case $70 \mathrm{~b}$, those with open squares case 70a, those with filled circles case 35b, those with crosses case 35a. Panel b): mass function plotted in redshift-independent form. The measured $f(\sigma)$ from all simulations in Table 1 of Tinker et al. (2008). Solid line is the best-fit function of Eq. (3) (Tinker et al. 2008). Dashed line indicates the model in the present paper.

numerical multiplicity functions (R03; YNY; W05; Del Popolo 2006a,b) and J01, are however within 1- $\sigma$ error bars, and so they agree overall. The multiplicity function obtained in the present paper peaks at $v \simeq 1$ as in YNY numerical multiplicity function, instead of a plateau as in the J01 function. Differences are also observed in the redshift evolution of the J01 mass function 
(Del Popolo 2006b). Fitting formulas presented by J01 are accurate to $\simeq 10-20 \%$ (Tinker et al. 2008, T08). In our model, the mass function is given by Eqs. (3)-(5), which agrees perfectly with the T08 mass function, as shown in Fig. 1b. The accuracy of the mass function is, as in T08, of $\simeq 5 \%$ for $\Lambda \mathrm{CDM}$ models for the mass and redshift range of interest in this study. In this way the theoretical uncertainties in the mass function do not contribute significantly to the systematic error budget. In Fig. 1b, we plot the mass function for all of our outputs in the $f(\sigma)-\ln \left(\sigma^{-1}\right)$ plane. High values of $\ln \sigma^{-1}$ correspond to rare haloes of high redshift and/or high mass, while low values of $\ln \sigma^{-1}$ describe haloes of low-mass and redshift combinations. Figure $1 \mathrm{~b}$ shows the function $f(\sigma)$ measured for all simulations in Table 1 of T08.

As previously reported, one of the main problems with using the mass function to constrain cosmological parameters is that theoretical predictions provide the number density for clusters of a given mass, while the mass itself is never the directly observed quantity. One then needs relations connecting mass with other more easily obtainable quantities that can be used as a surrogate for cluster mass. Over the past decade, observations of clusters of galaxies (e.g. ROSAT, ASCA) have shown the existence of a correlation between the total gravitating mass of clusters, $M_{\text {tot }}{ }^{4}$, their X-ray luminosity $\left(L_{\mathrm{X}}\right)$, and the temperature $\left(T_{\mathrm{X}}\right)$ of the intracluster medium (ICM) (David et al. 1993; Markevitch 1998; Horner et al. 1999). By means of the quoted scaling relations, one can obtain different methods for tracing the evolution of the cluster number density: (1) the X-ray temperature function (XTF), which has been presented for local (e.g., Henry \& Arnaud 1991) and distant clusters (Eke et al. 1998; Henry 2000); and (2) the evolution of the XLF. In this case, we need a relation between the observed $L_{\mathrm{X}}$ and the cluster virial mass.

In the present paper, following M08, we use the XLF to constrain cosmological parameters. Then the next crucial step is to convert it in a XLF. This can be done by first converting mass into intracluster gas temperature, by means of the $M-T_{\mathrm{x}}$ relation, and then converting the temperature into X-ray luminosity, by means of the $L_{\mathrm{x}}-T_{\mathrm{x}}$ relation. M08 used a self-similar relationship between mass and X-ray luminosity for massive clusters (e.g., Bryan \& Norman 1998) modified by an additional redshiftdependent factor (see Morandi et al. 2007). At this point, we must stress that numerical simulations confirm that the DM component in clusters of galaxies, which represents the dominant fraction of the mass, has a remarkably self-similar behaviour, but the baryonic component does not show the same level of self-similarity. This picture is confirmed by X-ray observations, see, for instance, the deviation of the luminosity-temperature $(L-T)$ relation in clusters, which is steeper than the theoretical value predicted by the previous scenario. More precisely, until some years ago, the cluster structure was considered to be scale-free, which means that the global properties of clusters, such as halo mass, $L-T$ relationship, and X-ray luminosity would scale self-similarly (Kaiser 1986; Evrard \& Henry 1991). In particular, the gas temperature would scale with cluster mass as $T \propto M^{2 / 3}$ and the bolometric X-ray luminosity would scale with temperature as $L \propto T^{2}$ in the bremsstrahlung-dominated regime above $2 \mathrm{keV}$.

Studies following that of Kaiser (1986) show that the observed luminosity-temperature relation is closer to $L \propto T^{3}$ (e.g., Edge \& Stewart 1991), indicating that non-gravitational

\footnotetext{
4 Since $M_{\text {tot }}$ compares with the ICM temperature measurements that can be obtained through X-ray spectroscopy, this explains the importance of a $M-T$ relation.
}

processes should influence the density structure of clusters core, where most of the luminosity is generated (Kaiser 1991; Evrard \& Henry 1991; Navarro et al. 1995; Bryan \& Norman 1998). One way to obtain a scaling law closer to the observational one is either to inject non-gravitational energy into the ICM before or during cluster formation, the so-called pre-heating (Ponman et al. 1999; Bower et al. 1997; Cavaliere et al. 1997, 1999; Tozzi \& Norman 2001; Borgani et al. 2001; Voit \& Brian 2001) or to consider feedback processes that alter the gas characteristics during the evolution of the cluster's (Voit \& Bryan 2001) cooling flows (Allen \& Fabian 1998). A similar situation is valid for the $M-T$ relationship, namely that the self-similarity in the $M-T$ relation seems to break at a few $\mathrm{keV}$ (Nevalanien et al. 2000; Xu et al. 2001; Finoguenov et al. 2001; Muanwong et al. 2001; Bialek et al. 2001). Consequently, if one starts with selfsimilar scaling laws, as in M08, in order to have consistent scaling relations one has to compare the self-similar scaling relations to observations (Morandi et al. 2007).

Unlike the M08 approach, we used models for the $L-T$, $M-T$, relationships taking the non-self similarity into account: namely, the $M-T_{\mathrm{x}}$ relation obtained analytically using the model of Del Popolo (2002), while the $L_{\mathrm{x}}-T_{\mathrm{x}}$ relation is the one obtained in Del Popolo et al. (2005) based on an improvement of the punctuated equilibrium model (PEM) of Cavaliere et al. (1997, 1998, 1999). The drawbacks of using self-similar relationships fitted to the data (clusters) and the reasons for using a different approach were already discussed in Del Popolo (2003, their Sect. 3), as well as in this section.

Similar to the 2009 study, in Del Popolo (2003) we used the models for the $L-T, T-M$, relationships instead of the scaling relations obtained from simulations of Chandra data (see, e.g., Pierpaoli et al. 2001, 2003, for references) ${ }^{5}$. Equation (5) in M08, similar to that Eq. (13) of Pierpaoli et al. (2001) or Eq. (4) of Pierpaoli et al. (2003), comes from rather simplistic arguments (dimensional analysis and an assumption that clusters are self-similar), not taking important physical effects into account, which then gives rise to a non-self-similar behaviour of the quoted relation, as previously discussed. The fitting procedure used by M08, trying to consider the previous physics and the non-self-similar behaviour of the relationship is complicated by several effects. In fact, in the fit one uses data that may contain small groups that can be influential in estimating the slope of the model, and one has then to carefully choose the data to be used in the fit. This choice mitigates the possibility of obtaining biased results if the slope of the mass-luminosity relation is different for massive clusters than for smaller groups. In M08 the data (clusters) were fitted with $L>3 \times 10^{44} \mathrm{erg} / \mathrm{s}$ in the $0.1-2.4 \mathrm{keV}$ band. Moreover, the process of fitting the model in Eq. (7) of M08 is complicated by the Malmquist bias. Close to the flux limit for selection, any X-ray-selected sample will tend to include the most luminous sources for a given mass. This results in a steepening of the derived mass-luminosity relation and a bias in the inferred intrinsic scatter in luminosity for a given mass. The use of the extended sample of Reiprich \& Böringer (2002) (RB02), rather than only their flux-limited HIFLUGCS sample, partially mitigates this effect by decreasing the flux limit. Another problem is that as a consequence of Malmquist bias there is a strong apparent, but not necessarily physical, correlation between luminosity and redshift because the flux limit corresponds to higher luminosities at higher redshifts.

\footnotetext{
5 Equation (22) in Del Popolo (2003) and Eq. (5) in M08 are very similar.
} 
For what concerns the data (clusters) used in the analysis, they are the same as those used by M08: the following three fluxlimited surveys are included in our analysis: the BCS (Ebeling et al. 1998) and REFLEX sample (Böhringer et al. 2004) at low redshifts $(z<0.3)$, and the MACS (Ebeling et al. 2001) at $0.3<z<0.5$ (see M08). In the analysis, the sample was chosen to cover the redshift range $z<0.5$, since at higher redshifts the number of unrelaxed clusters decrease, and the $L-T$ and $T-M$ relations are appropriate for relaxed clusters. In this paper we present an analysis based only on the XLF data described above, along with the priors described in Sect. 4 of M08.

Following M08, we parametrize the full model fitted to the XLF data as $h, \Omega_{\mathrm{b}} h^{2}, \Omega_{\mathrm{c}} h^{2}, \sigma_{8}, n_{\mathrm{s}}, w$, where $\Omega_{\mathrm{b}}$ and $\Omega_{\mathrm{c}}$ are the baryon and cold dark matter densities $\left(\Omega_{\mathrm{m}}=\Omega_{\mathrm{b}}+\Omega_{\mathrm{c}}\right)$. In addition to the assumption of spatial flatness, we adopt the Gaussian priors $h=0.72 \pm 0.08$ (Freedman et al. 2001) and $\Omega_{\mathrm{b}} h^{2}=0.0214 \pm 0.002$ (Kirkman et al. 2003) from the Hubble Key Project and Big Bang nucleosynthesis studies, respectively. Since the results are insensitive to the spectral index within a reasonable range (see M08), we fix $n_{\mathrm{s}}=0.95$ in accordance with (Spergel et al. 2007) for the standard analysis. The dark-energy equation of state was bounded by a uniform prior, $-5<w<0$.

The luminosity function likelihood is the same as in M08 (Sect. 4.2). The likelihood that $N$ clusters with inferred luminosities in a range $\mathrm{d} \hat{L}$ exist in a volume $\mathrm{d} V$ can in general be written as a Poisson probability plus a correction for the clustering of haloes with one another. If the plane of redshift and inferred luminosity is divided into non-overlapping cells, then the likelihood of our data is simply

$P\left(N_{1}, N_{2}, \ldots\right)=\prod_{j} \frac{\tilde{N}_{j}^{N_{j}} \mathrm{e}^{-\tilde{N}_{j}}}{N_{j} !}$,

where $N_{j}$ and $\tilde{N}_{j}$ are the number of clusters detected and predicted in the $j$ th cell, respectively.

If the cells are taken to be rectangular, with the $j$ th cell given by $z_{j}^{(1)} \leq z<z_{j}^{(2)}$ and $\hat{L}_{j}^{(1)} \leq \hat{L}<\hat{L}_{j}^{(2)}$, then

$\tilde{N}_{j}=\int_{z_{j}^{(1)}}^{z_{j}^{(2)}} \mathrm{d} z \frac{\mathrm{d} V(z)}{\mathrm{d} z} \int_{\hat{L}_{j}^{(1)}}^{\hat{L}_{j}^{(2)}} \mathrm{d} \hat{L} \frac{\mathrm{d} \tilde{n}(z, \hat{L})}{\mathrm{d} \hat{L}}$

where $V(z)$ is the comoving volume within redshift $z$. In the absence of intrinsic scatter in the mass-luminosity relation and measurement errors in the observed luminosities, the derivative of the comoving number density would simply be

$\frac{\mathrm{d} \tilde{n}(z, L)}{\mathrm{d} L}=f_{\text {sky }}(z, L) \frac{\mathrm{d} M(L)}{\mathrm{d} L} \frac{\mathrm{d} n(z, M)}{\mathrm{d} M}$.

Here, $f_{\text {sky }}$ is the sky coverage fraction of the surveys as a function of redshift and inferred luminosity, $\mathrm{d} n / \mathrm{d} M$ is no longer the Jenkins mass function but now the one discussed in the present paper, and $M(L)$ is the mass-luminosity relation.

Similar to M08, the presence of scatter requires us to take into account that a cluster detected with inferred luminosity $\hat{L}$ could potentially have any true luminosity $L$ and mass $M$, with some associated probability. To calculate the predicted number density correctly, we must therefore convolve with these probability distributions:

$$
\begin{aligned}
\frac{\mathrm{d} \tilde{n}(z, \hat{L})}{\mathrm{d} \hat{L}}= & f_{\text {sky }}(z, \hat{L}) \int_{0}^{\infty} \mathrm{d} L P(\hat{L} \mid L) \\
& \times \int_{0}^{\infty} \mathrm{d} M P(L \mid M) \frac{\mathrm{d} n(z, M)}{\mathrm{d} M} .
\end{aligned}
$$

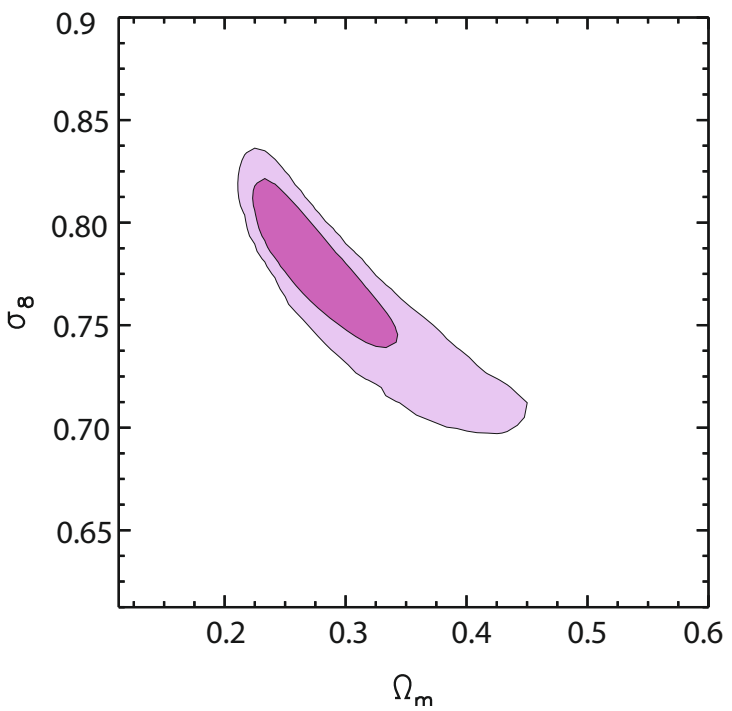

Fig. 2. Joint 68.3 and $95.4 \%$ confidence constraints on $\Omega_{\mathrm{m}}$ and $\sigma_{8}$ for a $\Lambda$ CDM model from the MACS, BCS, and REFLEX combination using standard priors (as described in the text).

where $P(L \mid M)$ is a log-normal distribution whose width is like in M08 the intrinsic scatter in the mass-luminosity relation, $\eta(z)$, and $P(\hat{L} \mid L)$ is a normal distribution whose width as a function of flux is modelled as a power law, as described in Sect. 3.2 of M08.

\section{Results}

In Fig. 2, we compare the joint $\Omega_{\mathrm{m}}-\sigma_{8}$ constraints obtained from the BCS, REFLEX, and MACS data set combination for a $\Lambda \mathrm{CDM}$. The marginalized constraints from the combination of the three cluster samples are $\Omega_{\mathrm{m}}=0.28_{-0.04}^{+0.05}$ and $\sigma_{8}=$ $0.78_{-0.05}^{+0.04}$, while the result of M08 gives less tight constraints $\Omega_{\mathrm{m}}=0.28_{-0.07}^{+0.11}$ and $\sigma_{8}=0.78_{-0.13}^{+0.11}$.

Our previous constraints are in good agreement with recent, independent results from the CMB (Spergel et al. 2007) and cosmic shear, as measured in the 100 Square Degree Survey (Benjamin et al. 2007) and CFHTLS (Canada-France-Hawaii Telescope Legacy Survey) Wide field (Fu et al. 2008). Our results also agree overall with previous findings based on the observed X-ray luminosity and temperature functions of clusters (Eke et al. 1998; Donahue \& Voit 1999; Henry 2000; Borgani et al. 2001; Seljak 2002; Allen et al. 2003; Pierpaoli et al. 2003; Schuecker et al. 2003; Henry 2004). Our result for $\Omega_{\mathrm{m}}$ is in excellent agreement with current constraints based on cluster $f_{\text {gas }}$ data (Allen et al. 2008, and references therein) and the power spectrum of galaxies in the $2 \mathrm{dF}$ galaxy redshift survey (Cole et al. 2005) and Sloan Digital Sky Survey (SDSS) (Eisenstein et al. 2005; Tegmark et al. 2006; Percival et al. 2007a), as well as the combination of CMB data with a variety of external constraints (Spergel 2007).

In Fig. 3a, we set constraints for the $w$ CDM model, and we plot the joint constraints on $\Omega_{\mathrm{m}}$ and $\sigma_{8}$ from the luminosity function data using our standard priors, while Fig. 3 b displays constraints on $\Omega_{\mathrm{m}}$ and $w$ obtained independently of the XLF data. The marginalized results from the XLF data are $\Omega_{\mathrm{m}}=0.27_{-0.06}^{+0.07}$, $\sigma_{8}=0.81_{-0.06}^{+0.05}$ and $w=-1.3_{-0.4}^{+0.3}$, while in M08 they are again less tight $\Omega_{\mathrm{m}}=0.24_{-0.07}^{+0.15}, \sigma_{8}=0.85_{-0.20}^{+0.13}$ and $w=-1.4_{-0.7}^{+0.4}$. Our 

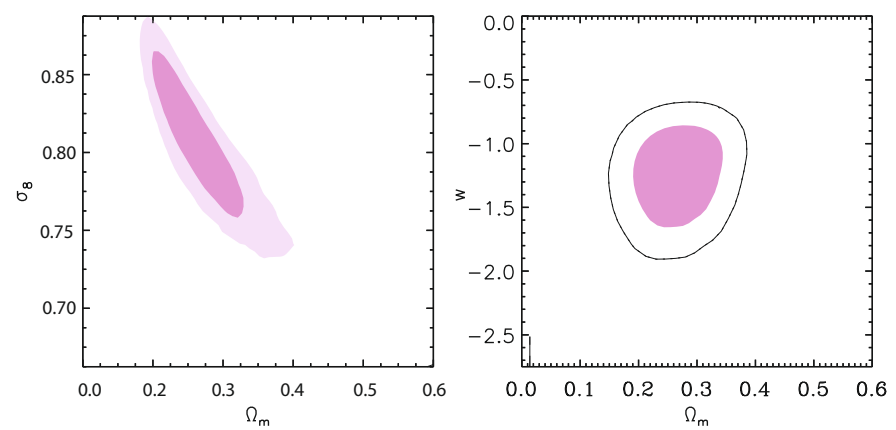

Fig. 3. Panel a). Same as Fig. 2 but for a $w$ CDM model. Panel b). Joint 68.3 and $95.4 \%$ confidence constraints on $\Omega_{\mathrm{m}}$ and $w$ for a constant- $w$ model using the XLF data and standard priors (as described in the text).
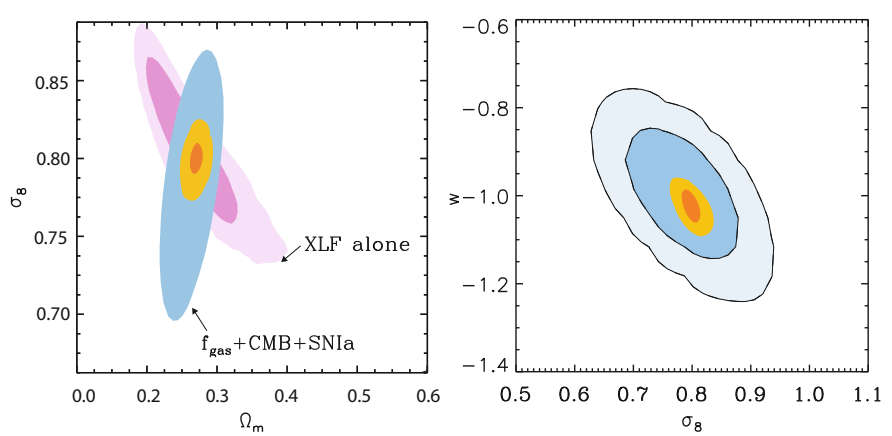

Fig. 4. Joint 68.3 and 95.4 per cent confidence constraints on $\Omega_{\mathrm{m}}$ and $\sigma_{8}$ (left panel) and $\sigma_{8}$ and $w$ (right panel) obtained from a combined $f_{\text {gas }}+\mathrm{CMB}+\mathrm{SNIa}$ analysis (blue) and the improved constraints obtained by combining these data with the XLF (gold). No priors on $h, \Omega_{\mathrm{b}} h^{2}$ or $n_{\mathrm{s}}$ are imposed in either analysis. In the left panel, the results from the XLF alone using standard priors are shown (purple) to illustrate the degeneracy breaking. In the left panel we plotted just the inner confidence contours in the $f_{\text {gas }}+\mathrm{CMB}+\mathrm{SNIa}$ analysis, to have a more readable plot.

new XLF results are consistent with the cosmological-constant model $(w=-1)$.

An improvement in the previous results can be obtained by adding to the XLF analysis the $f_{\text {gas }}+\mathrm{CMB}+\mathrm{SNIa}$ data set. The $f_{\text {gas }}+\mathrm{CMB}+\mathrm{SNIa}$ combination already provides tight constraints on $\Omega_{\mathrm{m}}, h, \Omega_{\mathrm{b}} h^{2}$, and $n_{\mathrm{s}}$ (so that no priors on these parameters are used in either of the combined analyses), but the degeneracy between $w$ and $\sigma_{8}$ (right panel of Fig. 4) limits the precision of the dark-energy results. The addition of the XLF data breaks the degeneracy in the $\Omega_{\mathrm{m}}-\sigma_{8}$ plane (left panel), resulting in tighter constraints on $\Omega_{\mathrm{m}}, \sigma_{8}$, and $w$. The degeneracy breaking power of other combinations of data with the CMB is discussed by Spergel et al. (2007). The resulting constraints are $\Omega_{\mathrm{m}}=0.269 \pm 0.012, \sigma_{8}=0.81 \pm 0.021$, and $w=$ $-1.02 \pm 0.04$, to be compared with M08 $\Omega_{\mathrm{m}}=0.269 \pm 0.016$, $\sigma_{8}=0.82 \pm 0.03$, and $w=-1.02 \pm 0.06$. The previous constraints agree within the errors with the Vikhlinin et al. (2009b) constraints, namely, $\Omega_{\mathrm{m}}=0.255 \pm 0.043, \sigma_{8}=0.786 \pm 0.011$, and $w=-0.991 \pm 0.045$.

The tight constraints obtained by M08 when combining XLF analysis with the $f_{\text {gas }}+\mathrm{CMB}+\mathrm{SNIa}$ data set are primarily due to the tightness of the constraints obtained from $f_{\text {gas }}+\mathrm{CMB}+\mathrm{SNIa}$ data itself and not to the precision of the XLF analysis of M08, as shown by comparing our results for $\Omega_{\mathrm{m}}, \sigma_{8}, w$ obtained by the
XLF analysis with those of M08. In our model the improvement in the mass function model and the $L-M$ relationship gives rise to tight constraints even when using only the XLF function.

To understand why the results of our analysis are different from those of M08, we have to stress a key point. The M08 paper, as well as several others papers in the literature, used two different data sets, the first one composed by REFLEX, BCS, and MACS, to constrain cosmological parameters and the second external data set (RB02 data set) to constrain the luminosity-mass relation, which in M08 is a power law with three free parameters, without explicitly accounting for selection bias. Consequently, it was necessary to restrict the external data set (RB02) to low redshifts and high fluxes to minimize the effects of selection bias, making it impossible to test for departures from self-similar evolution in the scaling relation. For a "self-consistent" analysis, it is necessary that a single likelihood function be applied to the full data set, which encompasses the entire theoretical model (cosmology + scaling relations) so as to ensure that the covariance among all the model parameters is fully captured and that the effects of the mass function and selection biases are properly accounted for throughout. This kind of analysis has been performed for the first time by Vikhlinin et al. (2009a,b), who used the same cluster sample to constrain the scaling relations, thus obtaining tighter constraints.

In the analysis of the present paper, the $L-M$ relation is a physically motivated relation (not a power law with free parameters) that does not require fits to data, as in M08. Since we do not need the double analysis of M08 and previous papers, the first to get the $L-M$ fitting parameters from RB02 data and the second to obtain the cosmological constraints using BCS, REFLEX, and MACS, we bypass the quoted drawback in the M08 analysis.

A month after the present paper was submitted, two papers, Mantz et al. (2009a,b), appeared in arXiv showing that the key point stressed previously, namely generalizing M08 to allow the quoted simultaneous and self-consistent fit and using T08 mass function (instead of that in Jenkins et al. 2001) result in cosmological constraints that are a factor 2-3 better than those in M08, based on the same flux-limited sample of clusters. In the present paper, we have also checked that using the same $L-M$ relation as in M08, we obtain the same set of constraints as derived by $\mathrm{M}^{6}{ }^{6}$ again.

The use of our non-self-similar $L-M$ relation for clusters of luminosity $L>3 \times 10^{44} \mathrm{erg} / \mathrm{s}$ in the $0.1-2.4 \mathrm{keV}$ band. Since the clusters included in the M08 sample have high X-ray luminosity (above $3 \times 10^{44} \mathrm{erg} / \mathrm{s}$ ), one could think that the changes in the $L-M$ relation of the present paper with respect to the classical self-similar model will not produce significant changes in constraints on the cosmological parameters. Even if major differences between the $L-M$ model of the present paper and the self-similar model are observed at gas temperatures below $3 \mathrm{keV}$, the present $L-M$ relation depends on the $M-T$ and $L-T$ relationships, and especially the second one (based on the modified punctuated equilibrium model (MPEM)) never behaves in a self-similar way as shown in Del Popolo et al. (2005, even at gas temperatures higher than $10 \mathrm{keV}$ ). Moreover, as previously reported, the improvement in the constraints is strictly connected bypassing the quoted drawback in the M08 analysis by means of our $L-M$ relation and does not depend on parameters that must be fixed using external data.

To obtain tighter and tighter constraints, one needs to try to reduce the systematic uncertainties in the analysis to a minimum.

\footnotetext{
${ }^{6}$ Mantz et al. (2009a,b) obtain $\Omega_{\mathrm{m}}=0.27 \pm 0.02, \sigma_{8}=0.79 \pm 0.03$ and $w=-0.96 \pm 0.06$.
} 
Progress is expected over the coming years in refining the ranges of these allowances, both observationally and through improved simulations. A reduction in the size of the required systematic allowances will tighten the cosmological constraints. Improved numerical simulations of large samples of massive clusters, including a more complete treatment of star formation and feedback physics that reproduces both the observed optical galaxy luminosity function and cluster X-ray properties, will be of major importance. Further deep X-ray and optical observations of nearby clusters will provide better constraints on the viscosity of the cluster gas. Improved optical/near infrared observations of clusters should pin down the stellar mass fraction in galaxy clusters and its evolution. Ground and space-based gravitational lensing studies will provide strong, independent constraints on the mass distributions in clusters. A large programme using the Subaru telescope and Hubble Space Telescope is underway, as is similar work by other groups (e.g. Hoekstra 2007).

In the near future, continuing programmes of Chandra and XMM-Newton observations of known X-ray luminous clusters should allow progress, both by expanding the $f_{\text {gas }}$ sample (e.g. Chandra snapshot observations of the entire MACS sample; Ebeling et al. 2001, 2007) and through deeper observations of the current target list. A new, large-area X-ray survey such as proposed by the Spectrum-RG/eROSITA project could make a substantial contribution by finding hundreds of suitable systems at high redshifts.

Looking a decade ahead, the International X-ray Observatory (IXO), the result of the merging of NASA's Constellation-X and ESA/JAXA's XEUS mission concepts, will offer the possibility to carry out precise studies of dark energy using the $f_{\text {gas }}$ technique .

\section{Conclusions}

We showed how to improve the constraints on $\Omega_{\mathrm{m}}, \sigma_{8}$, and the dark-energy equation-of-state parameter $w$, from measurements of the XLF of galaxy clusters, as performed by M08. Improving the mass function by means of the Del Popolo (2006a,b) model, which was shown to be in good agreement with T08, and using the $L-M$ relationship obtained in Del Popolo (2002) and Del Popolo et al. (2005), we showed that the XLF alone can give tight constraints on the cosmological parameters. Using the same methods and priors as in M08, we find $\Omega_{\mathrm{m}}=0.28_{-0.04}^{+0.05}$ and $\sigma_{8}=0.78_{-0.05}^{+0.04}$ for a $\Lambda \mathrm{CDM}$ universe, and similarly in the case of a $\omega \mathrm{CDM}$ model, we find $\Omega_{\mathrm{m}}=0.27_{-0.06}^{+0.07}, \sigma_{8}=0.81_{-0.06}^{+0.05}$, and $w=-1.3_{-0.4}^{+0.3}$, both tighter than the M08 results. Combining the XLF analysis with the $f_{\text {gas }}+\mathrm{CMB}+\mathrm{SNIa}$ data set results in the constraint $\Omega_{\mathrm{m}}=0.269 \pm 0.012, \sigma_{8}=0.81 \pm 0.021$, and $w=-1.02 \pm 0.04$, in agreement with the most recent determination of the quoted parameters (Allen et al. 2008; Vikhlinin et al. 2009b; Percival et al. 2007b). Our findings, consistent with $w=-1$, lends additional support to the cosmological-constant model.

\footnotetext{
7 The large collecting area and combined spatial/spectral resolving power of IXO should permit precise $f_{\text {gas }}$ measures. An investment of $\simeq 10 \mathrm{Ms}$ of IXO time to measure $f_{\text {gas }}$ to $5 \%$ (corresponding to $3.3 \%$ accuracy in distance) in each of the 500 hottest, most X-ray luminous, dynamically relaxed clusters detected in future cluster surveys, spanning the redshift range $0<z<2$ (typical redshift $z \simeq 0.6$ ), will be sufficient to constrain cosmological parameters with a DETF figure of merit of 2040.
}

Acknowledgements. The author acknowledges financial support from the German Research Foundation (DFG) under grant NO. KR 1635/16-1.

\section{References}

Allen, S. W., \& Fabian, A. 1998, MNRAS, 297, L57

Allen, S. W., Schmidt, R. W., Fabian, A. C., \& Ebeling, H. 2003, MNRAS, 342, 287

Allen, S. W., Rapetti, D. A., Schmidt, R. W., et al. 2008, MNRAS, 383, 879 Bahcall, N. A., \& Fan, X. 1998, ApJ, 504, 1

Bahcall, N. A., Fan, X., \& Cen, R. 1997, ApJ, 485, L53

Bardeen, J. M., Bond, J. R., Kaiser, N., \& Szalay, A. S. 1986, ApJ, 304, 15

Benjamin, J., Heymans, C., Semboloni, E., et al. 2007, MNRAS, 381, 702

Bialek, J. J., Evrard, A. E., \& Mohr, J. J. 2001, ApJ, 555, 597

Blanchard, A., \& Bartlett, J. G. 1998, A\&A, 332, L49

Blanchard, A., Bartlett, J. G., \& Sadat, R. 1999, CRASB, 327, 313

Böhringer, H., Schuecker, P., Guzzo, L., et al. 2004, A\&A, 425, 367

Borgani, S., Rosati, P., Tozzi, P., et al. 2001, ApJ, 561, 13

Bower, R. G., Castander, F. J., Couch, W., Ellis, R. S., \& Böhringer, H. 1997, MNRAS, 291, 353

Bryan, G. L., \& Norman, M. L. 1998, ApJ, 495, 80

Burenin, R. A., Vikhlinin, A., Hornstrup, A., et al. 2007, ApJS, 172, 561

Cavaliere, A., Menci, N., \& Tozzi, P. 1997, ApJ, 484, L21 (CMT97)

Cavaliere, A., Menci, N., \& Tozzi, P. 1998, ApJ, 501, 493 (CMT98)

Cavaliere, A., Menci, N., \& Tozzi, P. 1999, MNRAS, 308, 599 (CMT99)

Cash, A. 1979, ApJ, 228, 939

Cole, S., Percival, W. J., Peacock, J. A., et al. 2005, MNRAS, 362, 505

David, L. P., Slyz, A., Jones, C., Forman, W., \& Vrtilek, S. D. 1993, ApJ, 412, 479

Del Popolo, A. 2002, MNRAS, 336, 81

Del Popolo, A. 2003, ApJ, 599, 723

Del Popolo, A. 2006a, ApJ, 637, 12

Del Popolo, A. 2006b, AJ, 131, 2367

Del Popolo, A., Hiotelis, S., \& Peńarrubia, G. 2005, ApJ, 628, 76

Donahue, M., \& Voit, G. M. 1999, ApJ, 523, L137

Dunkley, J., Spergel, D. N., Komatsu, E., et al. 2009, ApJ, 701, 1804

Ebeling, H., Edge, A. C., Bohringer, H., et al. 1998, MNRAS, 301, 881

Ebeling, H., Edge, A. C., Allen, S. W., et al. 2000, MNRAS, 318, 333

Ebeling, H., Edge, A. C., \& Henry, J. P. 2001, ApJ, 553, 668

Ebeling, H., Barrett, E., Donovan, D., et al. 2007, ApJ, 661, L33

Edge, A. C., \& Stewart, G. C. 1991, MNRAS, 252, 414

Eke, V. R., Cole, S., \& Frenk, C. S. 1996, MNRAS, 282, 263

Eke, V. R., Cole, S., Frenk, C. S., \& Patrick Henry, J. 1998, MNRAS, 298, 1145

Eisenstein, D. J., Zehavi, I., Hogg, D. W., et al. 2005, ApJ, 633, 560

Finoguenov, A., Reiprich, T. H., \& Böhringer, H. 2001, A\&A, 368, 749

Freedman, W. L., McClintock, J. E., Narayan, R., et al. 2001, ApJ, 553, 47

Fu, L., Semboloni, E., Hoekstra, H., et al. 2008, A\&A, 479, 9

Gao, L., Navarro, J. F., Cole, S., et al. 2007, MNRAS, 387, 536

Governato, F., Babul, A., Quinn, T., et al. 1999, MNRAS, 307, 949

Gregory, P. C., \& Loredo, T. 1992, ApJ, 398, 146

Henry, J. P. 2000, ApJ, 534, 565

Henry, J. P. 2004, ApJ, 609, 603

Henry, J. P., \& Arnaud, K. A. 1991, ApJ, 372, 410

Horner, D. J., Mushotzky, R. F., \& Scharf, C. A. 1999, ApJ, 520, 78

Hoekstra, H. 2007, MNRAS, 379, 317

Hu, W., \& Kravtsov, A. V. 2003, ApJ, 584, 702

Jenkins, A., Frenk, C. S., White, S. D. M., et al. 2001, MNRAS, 321, 372

Jeltema, T. E., Hallman, E. J., Burns, J. O., \& Motl, P. M. 2008, ApJ, 681, 167

Kaiser, N. 1986, MNRAS, 222, 323

Kaiser, N. 1991, ApJ, 383, 104

Kirkman, D., Tytler, D., Suzuki, N., O’Meara, J. M., \& Lubin, D. 2003, ApJS, 149, 1

Komatsu, E., Dunkley, J., Nolta, M. R., et al. 2009, ApJS, 180, 330

Kravtsov, A. V., Vikhlinin, A., \& Nagai, D. 2006, ApJ, 650, 128

Maughan, B. J. 2007, ApJ, 668, 772

Mantz, A., Allen, S. W., Ebeling, H., \& Rapetti, D. 2008, MNRAS, 387, 1179 (M08)

Mantz, A., Allen, S. W., Rapetti, D., \& Ebeling, H. 2009a [arXiv: 0909. 3098]

Mantz, A., Allen, S. W., Ebeling, H., et al. 2009b [arXiv: 0909. 3099]

Markevitch, M. 1998, ApJ, 503, 77

Morandi, A., Ettori, S., \& Moscardini, L. 2007, MNRAS, 379, 518

Muanwong, O., Thomas, P. A., Kay, S. T., Pearce, F. R., \& Couchman, H. M. P. 2001, ApJ, 552, L27

Nagai, D., Vikhlinin, A., \& Kravtsov, A. V. 2007, ApJ, 655, 98

Navarro, J. F., Frenk, C. S., \& White, S. D. M. 1995, MNRAS, 275, 720

Nevalainen, J., Markevitch, M., \& Forman, W. 2000, ApJ, 532, 694

Percival, W. J., Nichol, R. C., Eisenstein, D. J., et al. 2007a, ApJ, 657, 51 
Percival, W. J., Cole, S., Eisenstein, D. J., et al. 2007b, MNRAS, 381, 1053 Pierpaoli, E., Scott, D., \& White, M. 2001, MNRAS, 325, 77

Pierpaoli, E., Borgani, S., Scott, D., \& White, M. 2003, MNRAS, 342, 163

Ponman, T. J., Cannon, D. B., \& Navarro, J. F. 1999, Nature, 397, 135

Press, W., \& Schechter, P. 1974, ApJ, 187, 425

Rasia, E., Ettori, S., Moscardini, L., et al. 2006, MNRAS, 369, 2013

Reed, D., Gardner, J., Quinn, T., et al. 2003, MNRAS, 346, 565 (R03)

Reiprich, T. H., \& Böhringer, H. 2002, ApJ, 567, 716

Reichart, D. E., Nichol, R. C., Castander, F. J., et al. 1999b, ApJ, 518, 521

Rosati, P., Borgani, S., \& Norman, C. 2002, ARA\&A, 40, 539

Sadat, R., Blanchard, A., \& Oukbir, J. 1998, A\&A, 329, 21

Schuecker, P., Böhringer, H., Collins, C. A., \& Guzzo, L. 2003, A\&A, 398, 867

Seljak, U. 2002, MNRAS, 337, 769

Sheth, R. K., \& Tormen, G. 2002, MNRAS, 329, 61

Spergel, D. N., Bean, R., Doré, O., et al. 2007, ApJS, 170, 377

Tegmark, M., Eisenstein, D. J., Strauss, M. A., et al. 2006, Phys. Rev. D, 74, 123507
Tinker, J., Kravtsov, A. V., Klypin, A., et al. 2008, ApJ, 688, 709

Tozzi, P., \& Norman, C. 2001, ApJ, 546, 63

Trümper, J. 1993, Science, 260, 1769

Viana, P. T. P., \& Liddle, A. R. 1999, MNRAS, 303, 535

Voit, C. M. 2000, ApJ, 543, 113

Vikhlinin, A., McNamara, B. R., Forman, W., et al. 1998, ApJ, 502, 558

Vikhlinin, A., Burenin, R. A., Ebeling, H., et al. 2009a, ApJ, 692, 1033

Vikhlinin, A., Kravtsov, A. V., Burenin, R. A., et al. 2009b, ApJ, 692, 1060

Voevodkin, A., \& Vikhlinin, A. 2004, ApJ, 601, 610

Voit, C. M., \& Donahue, M. 1998, ApJ, 500, L111

Voit, G. M., \& Bryan, G. 2001, Nature, 414, 425

Warren, M. S., Abazajian, K., Holz, D. E., \& Teodoro, L. 2006, ApJ, 646, 881

White, S. D. M., Navarro, J. F., Evrard, A. E., \& Frenk, C. S. 1993, Nature, 366, 429

Xu, H., Jing, G., \& Wu, X. 2001, ApJ, 553, 78

Yahagi, H., Nagashima, M., \& Yoshii, Y. 2004, ApJ, 605, 709 (YNY04)

Zhang, Y. Y., Finoguenov, A., Böhringer, H., et al. 2007, A\&A, 467, 437 